\title{
Configuration optimization of UV reactors for water disinfection with computational fluid dynamics: Feasibility of using particle minimum UV dose as a performance indicator
}

\author{
Wentao Li ${ }^{a}$, Mengkai Li ${ }^{\text {a }}$, James R. Bolton ${ }^{\mathrm{b}}$, Zhimin Qiang ${ }^{\mathrm{a}, *}$ \\ ${ }^{a}$ Key Laboratory of Drinking Water Science and Technology, Research Center for Eco-Environmental Sciences, University of Chinese Academy of Sciences, Chinese Academy of \\ Sciences, 18 Shuang-qing Road, Beijing 100085, China \\ ${ }^{\mathrm{b}}$ Department of Civil and Environmental Engineering, University of Alberta, Edmonton, AB T6G 2W2, Canada
}

\section{H I G H L I G H T S}

- $D_{\min }$ was used as a performance indicator for UV reactor configuration optimization.

- $D_{\min }$ proved to be a qualified alternative to RED for UV reactor optimal design.

- Reactor with a well-mixed flow dispersed by lamp array had the best performance.

- Disinfection capability of the optimally designed UV reactor was elevated by $47-100 \%$.

\section{A R T I C L E I N F O}

Article history:

Received 1 April 2016

Received in revised form 14 June 2016

Accepted 10 July 2016

Available online 11 July 2016

Keywords:

UV reactor

Water disinfection

Configuration optimization

Performance indicator

Particle minimum UV dose
G R A P H I C A L A B S T R A C T

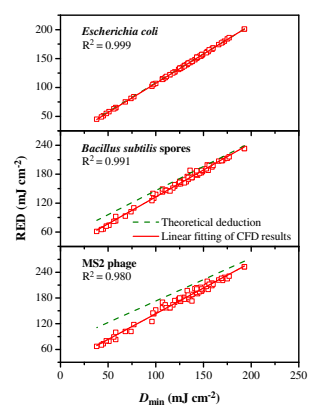

\begin{abstract}
A B S T R A C T
Computational fluid dynamics is a powerful tool for ultraviolet (UV) reactor performance evaluation, since it allows the reactor configuration optimization by comparing the performance indicator [e.g., reduction equivalent dose (RED)] of various UV reactors. In this study, a new performance indicator independent of the microbial UV dose-response curve, namely particle minimum UV dose $\left(D_{\min }\right)$, was introduced, and its feasibility was evaluated in the configuration optimization of 3-lamp UV reactors for water disinfection. Results indicate that the $D_{\min }$ could serve as an alternative to the RED in characterizing the UV reactor performance. In general, reactors with a normal (NOR) lamp position pattern (i.e., with one lamp on the top and two at the bottom) had larger $D_{\min }$ values $\left(110-193 \mathrm{~mW} \mathrm{~cm}^{-2}\right)$ than those with a reverse (REV) lamp position pattern (i.e., with two lamps on the top and one at the bottom) $\left(98-160 \mathrm{~mW} \mathrm{~cm}^{-2}\right)$. The largest $D_{\min }\left(193 \mathrm{~mW} \mathrm{~cm}^{-2}\right)$ was obtained in an UV reactor with a lamp relative distance (i.e., the ratio of the distance between the lamp and reactor axes to the reactor radius) of 0.4 and an inlet internal diameter of $75 \mathrm{~mm}$, which could be ascribed to a well-mixed flow resulting from an optimal dispersion of the influent flow by the lamp array. The maximum disinfection flow rate of the UV reactor with the optimal configuration was elevated by $47-100 \%$ compared to those of the non-optimally designed reactors, which led to an energy saving efficiency of $32-50 \%$.

() 2016 Elsevier B.V. All rights reserved.
\end{abstract}

\footnotetext{
* Corresponding author.

E-mail address: qiangz@rcees.ac.cn (Z. Qiang).
}

\section{Introduction}

The application of ultraviolet (UV) technology in drinking water treatment is increasing rapidly worldwide because of its cost- 


\section{Nomenclature}

$D_{\text {avg }} \quad$ particle average UV dose $\left(\mathrm{mJ} \mathrm{cm}^{-2}\right)$

$D_{\mathrm{c}} \quad$ assumed particle constant UV dose $\left(\mathrm{mJ} \mathrm{cm}^{-2}\right)$

$D_{\text {min }} \quad$ particle minimum UV dose $\left(\mathrm{mJ} \mathrm{cm}^{-2}\right)$

$D_{\mathrm{i}} \quad$ UV dose received by the $i^{\text {th }}$ particle $\left(\mathrm{mJ} \mathrm{cm}^{-2}\right)$

$D_{0} \quad$ intercept of microbial UV dose-response curve on $\mathrm{X}$ axis $\left(\mathrm{mJ} \mathrm{cm} \mathrm{cm}^{-2}\right)$

$D_{10} \quad$ particle $10^{\text {th }}$ percentile UV dose $\left(\mathrm{mJ} \mathrm{cm}^{-2}\right)$

$d_{\mathrm{r}} \quad$ lamp relative distance $(-)$

$d_{1}$ reactor internal diameter $(\mathrm{mm})$

$d_{2} \quad$ reactor inlet or outlet internal diameter $(\mathrm{mm})$

FR fluence rate $\left(\mathrm{mW} \mathrm{cm}^{-2}\right)$

$\mathrm{FR}_{\mathrm{avg}}$ area-average fluence rate $\left(\mathrm{mW} \mathrm{cm}^{-2}\right)$

ID internal diameter ( $\mathrm{mm})$

$k$ microbial UV inactivation rate constant $\left(\mathrm{cm}^{2} \mathrm{~mJ}^{-1}\right)$

$L_{1} \quad$ reactor length $(\mathrm{mm})$
$L_{2} \quad$ lamp arc length ( $\mathrm{mm}$ )

$L_{3} \quad$ distance from reactor inlet to outlet $(\mathrm{mm})$

$N \quad$ total simulating particle number $(-)$

Q flow rate $\left(\mathrm{m}^{3} \mathrm{~h}^{-1}\right)$

$Q_{\max } \quad$ maximum disinfection flow rate $\left(\mathrm{m}^{3} \mathrm{~h}^{-1}\right)$

RED reduction equivalent dose $\left(\mathrm{mJ} \mathrm{cm}^{-2}\right)$

RSD relative standard deviation $(-)$

$r \quad$ reactor radius $(\mathrm{mm})$

$T_{\text {avg }} \quad$ particle average retention time (s)

$T_{\text {Dmin }} \quad$ particle retention time with the minimum UV dose (s)

$T_{10} \quad$ particle $10^{\text {th }}$ percentile retention time $(\mathrm{s})$

$x \quad$ distance between the lamp and reactor axes $(\mathrm{mm})$

$\theta_{10} \quad$ normalized particle $10^{\text {th }}$ percentile retention time (-) effectiveness in inactivating Cryptosporidium and Giardia [1] and great potential for micro-pollutant removal when used in combination with other chemicals [2,3]. Although UV disinfection has not yet been commonly adopted in drinking water treatment plants in China, its use for secondary water supply in communities with high-rise buildings has already become widespread owing to an encouraging policy [4]. The performance of a UV reactor for water disinfection (i.e., disinfection efficiency) depends on the fluence received by microorganisms while traveling through the reactor and is highly related to the radiation and flow fields inside the reactor chamber. In cases when the flow rate $(Q)$ and water UV transmittance are constant, the fluence rate (FR) distribution and hydrodynamics inside the reactor chamber are determined only by the reactor configuration. Hence, by comparing the effective fluences delivered by UV reactors with various configurations, one can sort out the optimal configuration with the largest effective fluence for a constant lamp power input, so as to increase the maximum disinfection flow rate $\left(Q_{\max }\right)$ and reduce the specific energy consumption as well.

The effective fluence of a UV reactor can be measured with biodosimetry [5], chemical dosimetry [6], or other suitable methods [7]; however, these measurements are practicable only for manufactured reactors, which are too costly to be applied for reactor design. Computational fluid dynamics (CFD) has recently been used to evaluate the UV reactor performance, and an integrated CFD simulation of UV disinfection reactors includes the simultaneous modeling of flow field, radiation field and microbial transportation and inactivation [8-9]. The behavior of microorganisms inside a UV reactor can be mimicked by releasing micro simulating particles at the reactor inlet, and the fluence received by each particle is calculated with a complied user-defined function [10]. Then, particle fluences can be converted to equivalent survival microbial concentrations using an appropriate microbial UV dose-response curve, and the accumulative survival microbial concentration at the reactor outlet is finally converted to an effective fluence which is known as the reduction equivalent fluence or reduction equivalent dose (RED) [10]. The microbial inactivation ratios or RED values of UV disinfection reactors predicted by CFD simulations have generally agreed with the measured results from biodosimetry [9-12].

The RED is a widely accepted performance indicator for UV reactors for water disinfection and the optimal reactor configuration can be obtained by comparing the RED values of UV reactors with various configurations. However, to obtain the RED, a specific UV dose-response curve of a certain challenge microorganism must be given at first and two conversions are needed as mentioned above. Besides, the RED depends highly on the microbial UV dose-response curve that is obtained from bench-scale microbial UV inactivation experiments with a quasi-collimated beam apparatus. Different RED values will be generated from the same simulation when using varied experimental microbial UV doseresponse curves [13]. Therefore, it is difficult to compare the results among studies that usually employ various microbial UV dose-response curves and sometimes even disparate challenge microorganisms. On that account, efforts have been made to search alternative performance indicators for UV disinfection reactors. Wols et al. [14] found a strong linear correlation $\left(R^{2}=0.99\right)$ between the disinfection efficiency (expressed in log-inactivation of microorganisms) and $D_{10}$ (the $10^{\text {th }}$ percentile dose in the UV dose distribution). Xu et al. [15] found that the disinfection efficiency was correlated to the normalized standard deviation of particle radial displacement. Nonetheless, all these findings were based on simulations with low corresponding RED values $\left(<50 \mathrm{~mJ} \mathrm{~cm}^{-2}\right)$. The target RED for reactor design will be considerably higher because both lamp aging and sleeve fouling have to be accounted for while still achieving a required minimum UV dose (i.e., $40 \mathrm{~mJ} \mathrm{~cm}^{-2}$ ) [16]. Other potential performance indicators include $\theta_{10}$ [17], $D_{\min }$ and $D_{\text {avg }}$ [14]. $\theta_{10}$ is the normalized particle $10^{\text {th }}$ percentile retention time, that is, the time when the first $10 \%$ of the micro simulating particles exit the reactor normalized by the theoretical mean retention time of all particles. $\theta_{10}$ has often been used as an indicator for short-circuiting in reactors [17]. $D_{\min }$ and $D_{\text {avg }}$ are the particle minimum UV dose and the particle average UV dose, respectively; which are the characteristic parameters of the UV dose distribution just like $D_{10}$ (Fig. S1). All these potential performance indicators have an advantage over the RED in being independent of the microbial UV dose-response curve. In addition, by evaluating their feasibilities in characterizing the UV disinfection reactor performance, one can determine the dominant parameter affecting the reactor performance so as to efficiently optimize the reactor configuration.

In this study, the configurations of 3-lamp UV reactors for water disinfection were optimized focusing on the lamp arrangement and the inlet size by using CFD simulations. In this optimization process, a new and simple performance indicator, $D_{\text {min }}$, was introduced, and its feasibility in characterizing the UV disinfection reactor performance was verified both theoretically and experimentally. The effects of the lamp arrangement and the inlet size on the reactor performance were clarified by analyzing the FR distribution and hydrodynamics, and the optimal configuration 

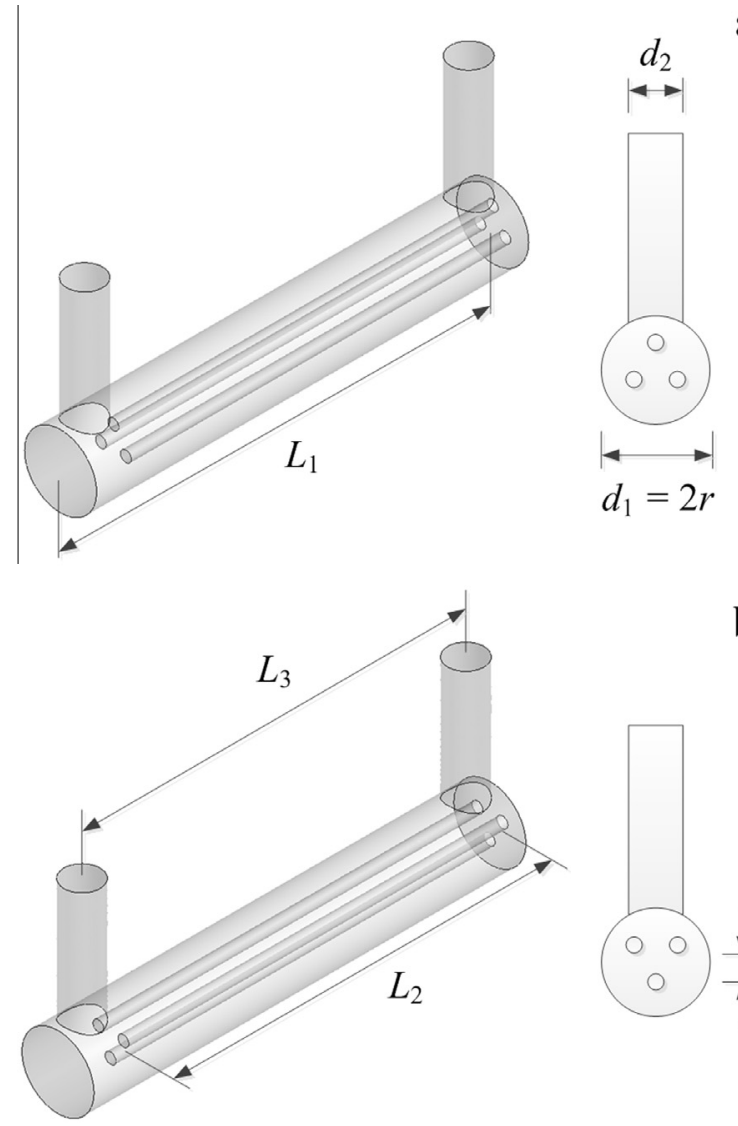

b REV

Fig. 1. Schematic diagram of the modeled UV disinfection reactors: (a) the NOR reactor, and (b) the REV reactor.

was decided by comparing the $D_{\text {min }}$ values of various reactors. The $Q_{\max }$ values of the reactors with or without the optimal configuration were also determined to assess the energy saving efficiency.

\section{Materials and methods}

\subsection{UV reactor configurations}

As illustrated in Fig. 1, annular UV reactors (U shape, that is, the inlet and outlet ports are located on the same side of the reactor) housing 3 low-pressure mercury vapor lamps were modeled in this study with a focus on various lamp arrangements and inlet sizes. The cross-sectional positions of the 3 lamps formed an equilateral triangle with its center located at the reactor axis. According to the lamp position pattern, the reactors were classified into two types: those with one lamp at the top (Fig. 1a) and those with two lamps at the top (Fig. 1b), which were denoted as the normal (NOR) and reverse (REV) lamp position reactors, respectively. The lamp relative distances $\left(d_{\mathrm{r}}\right)$, represented by the ratio of the distance between the lamp and reactor axes $(x)$ to the reactor radius $(r)$, were set at $0.4,0.5,0.6$ and 0.7 , respectively. The reactor length $\left(L_{1}\right)$ was $900 \mathrm{~mm}$ with an internal diameter (ID) $\left(d_{1}\right)$ of $150 \mathrm{~mm}$. The lamp arc length $\left(L_{2}\right)$ was $790 \mathrm{~mm}$ and the outside diameter of the lamp sleeve was $23 \mathrm{~mm}$. Each lamp had a power of $105 \mathrm{~W}$ with a UVC efficiency of $30 \%$. The inlet and outlet ID $\left(d_{2}\right)$ values were the same and 10 different sizes ranging from 30 to $150 \mathrm{~mm}$ were modeled. The distance from the inlet to the outlet $\left(L_{3}\right)$ was $800 \mathrm{~mm}$ when the inlet ID was not greater than $90 \mathrm{~mm}$, and $740 \mathrm{~mm}$ when the inlet ID was above. For simplicity, in the following sections the various reactor configurations were denoted in the form of NOR(or
REV)- $d_{\mathrm{r}}$-inlet ID (mm). For example, the NOR-0.4-75 reactor represented an NOR lamp position pattern, $d_{r}=0.4$, and inlet ID $=75 \mathrm{~mm}$.

\subsection{Model settings}

The modeling process was accomplished with a commercial CFD software package (ANSYS Fluent 13.0, Canonsburg, PA, USA). The computational domain of each UV reactor was discretized into more than 0.5 million small tetrahedral cells using the unstructured meshing method of patch conforming. The maximum mesh size was less than $0.01 \mathrm{~m}$, fine enough to produce mesh independent results, because further reducing the mesh size led to little difference $(<2 \%)$ of the simulation results. The Realizable $k-\varepsilon$ model was employed for the hydrodynamics modeling [18]. A uniformly distributed velocity was applied at the velocity inlet, whose value was determined based on the target flow rate and the inlet size. The FR distribution inside the reactor chamber was predicted with the discrete ordinates (DO) radiation model, with the UV light assumed to be emitted from the lamp sleeve [19]. The temperature of the computational domain was set close to zero ( $3 \mathrm{~K}$ in this study) to eliminate the interference of blackbody emission [20]. To mimic the movements of microorganisms flowing through the reactor, more than 5000 micro simulating particles were released at the inlet with their trajectories simulated using the Lagrangian approach of the discrete phase model. A compiled user-defined function was employed to calculate the UV dose received by each particle along its trajectory [10]. Convergence of each solution was obtained when the normalized residuals were all less than $10^{-3}$.

This set of CFD sub-models (i.e., Realizable $k-\varepsilon$ model, DO radiation model, and Lagrangian approach of the discrete phase model) has proven to produce reliable results with an average absolute error of less than 20\% [10]. Besides, although CFD simulations may predict a different level of change compared to experimental results when the UV reactor configuration varies, the trend in the reactor performance can still be correctly predicted [21]. Throughout this study the water UV transmittance was set at $90 \%$, a typical value for drinking water disinfection. In simulations for configuration optimization $(n=52), Q$ was kept constant at $3.53 \mathrm{~m}^{3} \mathrm{~h}^{-1}$; and in simulations for $Q_{\max }$ determination ( $n=18$ ), $Q$ varied from 3.53 to $15.90 \mathrm{~m}^{3} \mathrm{~h}^{-1}$.

\subsection{Calculation of performance indicators}

The RED was calculated off-line following the procedure proposed by Munoz et al. [22]. Assuming the UV dose-response of a challenge microorganism follows the delayed Chick-Watson model, the RED can be calculated as below:

$\mathrm{RED}=-\frac{1}{k} \lg \left(\frac{1}{N} \sum_{i=1}^{N} 10^{-k\left(D_{i}-D_{0}\right)}\right)+D_{0}$

where $k$ is the inactivation rate constant of the challenge microorganism $\left(\mathrm{cm}^{2} \mathrm{~mJ}^{-1}\right), D_{0}$ is the intercept of the UV dose-response curve on the $\mathrm{X}$ axis $\left(\mathrm{mJ} \mathrm{cm}^{-2}\right), N$ is the number of the total micro simulating particles, and $D_{\mathrm{i}}$ is the UV dose received by the $i^{\text {th }}$ particle $\left(\mathrm{mJ} \mathrm{cm}^{-2}\right)$. The challenge microorganism used for RED calculation in this study was Bacillus subtilis spores (ATCC 6633) unless otherwise specifically stated. According to our previous study, the values of $k$ and $D_{0}$ were determined to be $0.087 \mathrm{~cm}^{2} \mathrm{~mJ}^{-1}$ and $3.0 \mathrm{~mJ} \mathrm{~cm}^{-2}$, respectively [4]. Other two challenge microorganisms with various UV resistances, Escherichia coli (ATCC 11229) and MS2 phage (ATCC 15597-B1), were also employed to further examine the feasibility of the alternative performance indicator. Accord- 
ing to Hijnen et al. [1], the UV inactivation rate constants for E. coli and MS2 phage are 0.506 and $0.055 \mathrm{~cm}^{2} \mathrm{~mJ}^{-1}$, respectively.

The values of the potential performance indicators, including $\theta_{10}, D_{\text {min }}, D_{10}$ and $D_{\text {avg, }}$, were determined by statistically analyzing the retention times and the received UV doses of all micro simulating particles at the outlet of the UV reactor.

\section{Results and discussion}

\subsection{Evaluation of potential performance indicators}

To be a qualified performance indicator for UV disinfection reactors, the candidate parameter should have a strong linear correlation with the RED, a well-accepted reactor performance indicator. The correlations between the RED and the potential performance indicators (i.e., $\theta_{10}, D_{\text {min }}, D_{10}$ and $D_{\text {avg }}$ ) were analyzed with the CFD simulation results $(n=70)$, as shown in Fig. 2 . The poorest correlation was found between RED and $\theta_{10}$ with $\mathrm{R}^{2}=0.009$ (Fig. 2a), which could be ascribed to the fact that the UV reactor performance is dependent on both the radiation and flow fields, and a weaker short-circuiting (i.e., a larger $\theta_{10}$ ) does not necessarily result in a better reactor performance. The RED was linearly correlated with $D_{10}\left(\mathrm{R}^{2}=0.847\right)$ and $D_{\text {avg }}\left(\mathrm{R}^{2}=0.717\right)$ (Fig. 2c and d), but the strongest correlation was found with $D_{\min }$ $\left(\mathrm{R}^{2}=0.991\right)$ (Fig. $\left.2 \mathrm{~b}\right)$. This result is somewhat contradictory to what reported by Wols et al. [14]. They found a strong correlation between the disinfection efficiency and $D_{10}$, and thus inferred that $D_{10}$ was superior to $D_{\min }$ in characterizing the UV disinfection reactor performance. The stronger correlation between RED and $D_{\min }$ in this study could be ascribed to an increased contribution of $D_{\min }$ to RED in simulations with higher UV doses, as reflected in Eq. (1). In fact, most of the RED values in our configuration optimization process were greater than $100 \mathrm{~mJ} \mathrm{~cm}^{-2}$ considering the lamp aging, sleeve fouling and safety factors; while the previous work had lower RED values $\left(<50 \mathrm{~mJ} \mathrm{~cm}^{-2}\right)$ as calculated from the microorganism inactivation efficiencies [14]. Therefore, for the configura- tion optimization of UV disinfection reactors, $D_{\min }$ is a better performance indicator than $D_{10}$.

The linear correlation between RED and $D_{\text {min }}$ in simulations with high RED values can also be theoretically deduced. Assuming 10,000 micro simulating particles are released at the UV reactor inlet, and one of them receives a UV dose of $D_{\min }$ while all the rest have the same dose of $D_{\mathrm{c}}$. According to Eq. (1), the corresponding RED can be calculated as follows:

$\mathrm{RED}=-\frac{1}{k} \lg \left(\frac{1}{10000} 10^{-k\left(D_{\min }-D_{0}\right)}+\frac{9999}{10000} 10^{-k\left(D_{\mathrm{c}}-D_{0}\right)}\right)+D_{0}$

or

$\mathrm{RED}=-\frac{1}{k} \lg \left(10^{-4-k\left(D_{\min }-D_{0}\right)}+10^{-k\left(D_{\mathrm{c}}-D_{0}\right)}\right)+D_{0}$

When $D_{\mathrm{c}}$ is large enough, that is:

$10^{-k\left(D_{\mathrm{c}}-D_{0}\right)}<0.001 \times 10^{-4-k\left(D_{\min }-D_{0}\right)}$

or

$D_{\mathrm{c}}>\frac{7}{k}+D_{\min }$

the contribution of particles with a UV dose of $D_{c}$ to the RED can be omitted, and Eq. (3) can thus be simplified as follows:

$\mathrm{RED} \approx \frac{4}{k}+D_{\text {min }}$

In fact, about $90 \%$ of the micro simulating particles received a UV dose greater than $\left(7 / k+D_{\text {min }}\right)$ according to the UV dose distribution in this study. Therefore, the linear correlation between RED and $D_{\text {min }}$ expressed by Eq. (6) can be applied to the real scenes in an approximate way. The theoretically deduced correlations between RED and $D_{\min }$ were compared with the CFD simulation results with three various challenge microorganisms [i.e., $E$. coli $\left(k=0.506 \mathrm{~cm}^{2} \mathrm{~mJ}^{-1}[1]\right)$, B. subtilis spores $\left(k=0.087 \mathrm{~cm}^{2} \mathrm{~mJ}^{-1}[4]\right)$, and MS2 phage $\left.\left(k=0.055 \mathrm{~cm}^{2} \mathrm{~mJ}^{-1}[1]\right)\right]$. As shown in Fig. 3, the RED had a strong linear correlation with $D_{\min }\left(\mathrm{R}^{2} \geqslant 0.980\right)$ regard-
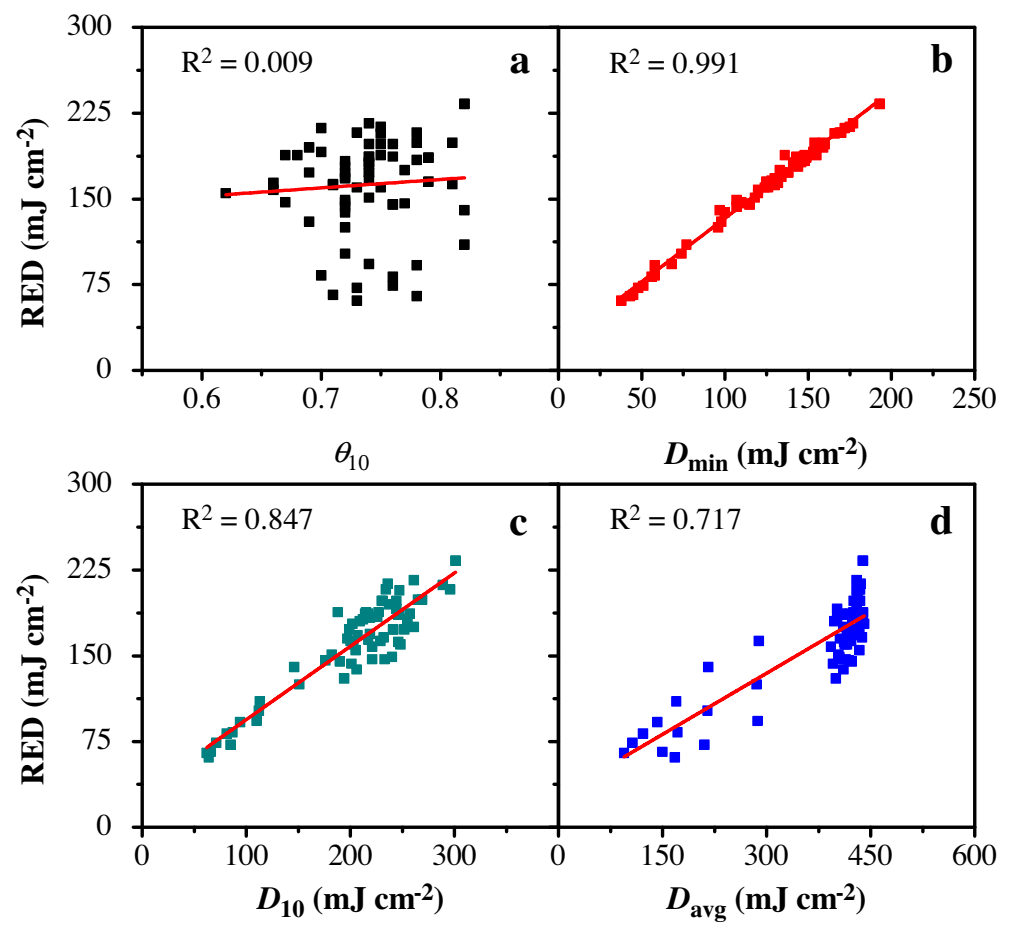

Fig. 2. Correlations between RED and the potential UV reactor performance indicators: (a) $\theta_{10}$, (b) $D_{\min }$, (c) $D_{10}$, and (d) $D_{\text {avg. }}$. 


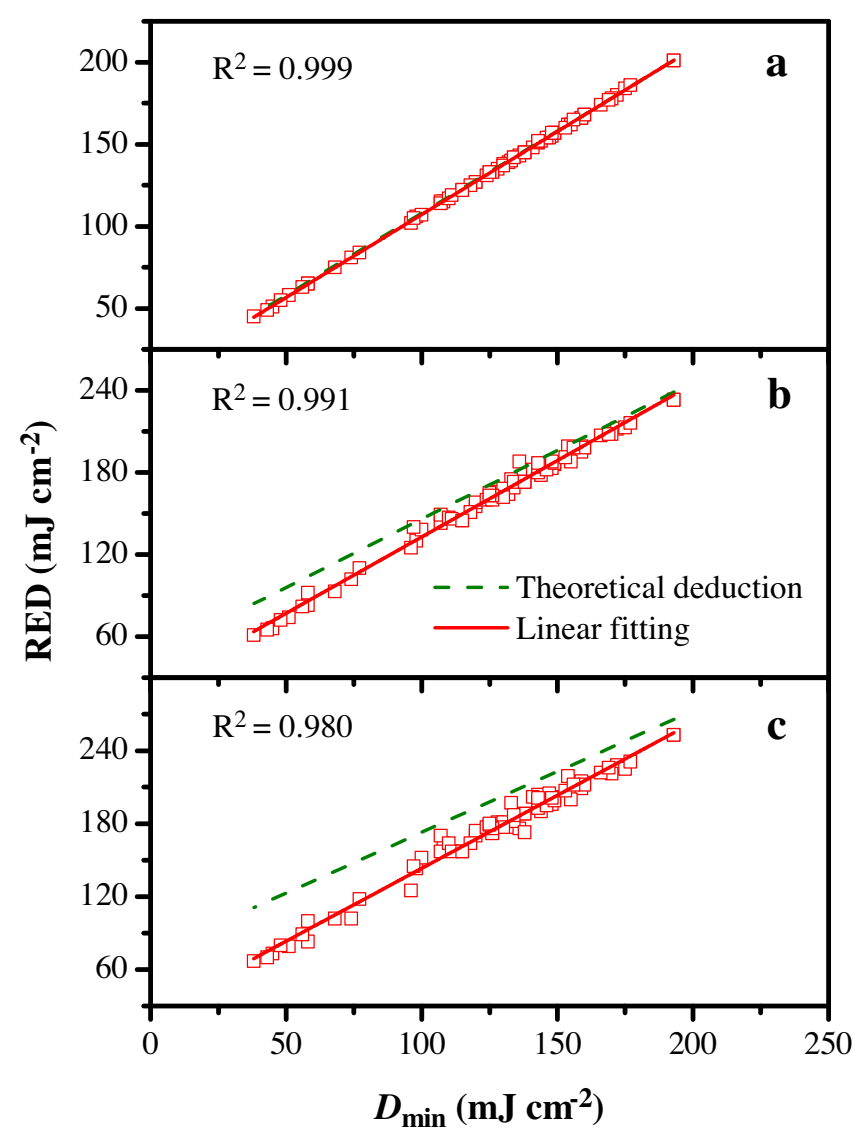

Fig. 3. Correlations between RED and $D_{\min }$ with various challenge microorganisms based on theoretical deductions (assuming a simplified UV dose distribution) and linear fittings of CFD simulation results: (a) Escherichia coli, (b) Bacillus subtilis spores, and (c) MS2 phage.

less of the challenge microorganisms, and the curves drawn based on the theoretical deductions lay close to the regression lines in all the three cases. The best agreement was found in the case with E. coli (Fig. 3a), which is reasonable because this case had the smallest $7 / k$ so that almost all particles $(>95 \%)$ fulfilled the condition of Eq. (5) and resulted in a scene very close to the assumptions for the theoretical deduction. This result confirmed the strong linear correlation between RED and $D_{\text {min }}$ both theoretically and experimentally. Therefore, $D_{\min }$ is a qualified alternative to the RED as a performance indicator for UV disinfection reactors, and a higher $D_{\min }$ indicates a better reactor performance.

\subsection{Effects of lamp relative distance and inlet ID on reactor performance}

The performances of the NOR UV reactors with various $d_{\mathrm{r}}$ values and inlet IDs as indicated by $D_{\mathrm{min}}$ are shown in Fig. 4 . Reactors with $d_{\mathrm{r}}=0.5$ had generally larger $D_{\min }$ values which varied from 124 to $175 \mathrm{~mJ} \mathrm{~cm}^{-2}$, while the smallest $D_{\text {min }}$ values were mostly found for reactors with $d_{\mathrm{r}}=0.7$ which ranged from 100 to $156 \mathrm{~mJ} \mathrm{~cm}^{-2}$. Contrast to other reactors whose $D_{\min }$ increased as the inlet ID approached $150 \mathrm{~mm}$, reactors with $d_{\mathrm{r}}=0.4$ reached the largest $D_{\min }$ $\left(193 \mathrm{~mJ} \mathrm{~cm}^{-2}\right)$ at an inlet ID of $75 \mathrm{~mm}$ (i.e., the NOR-0.4-75 reactor), which was also the largest value among all the reactors modeled.

The different performances of UV reactors with various configurations arose from the differences in FR distribution and hydrodynamics. Table 1 shows the characteristic parameters for FR distributions in the central cross sections of UV reactors with var-

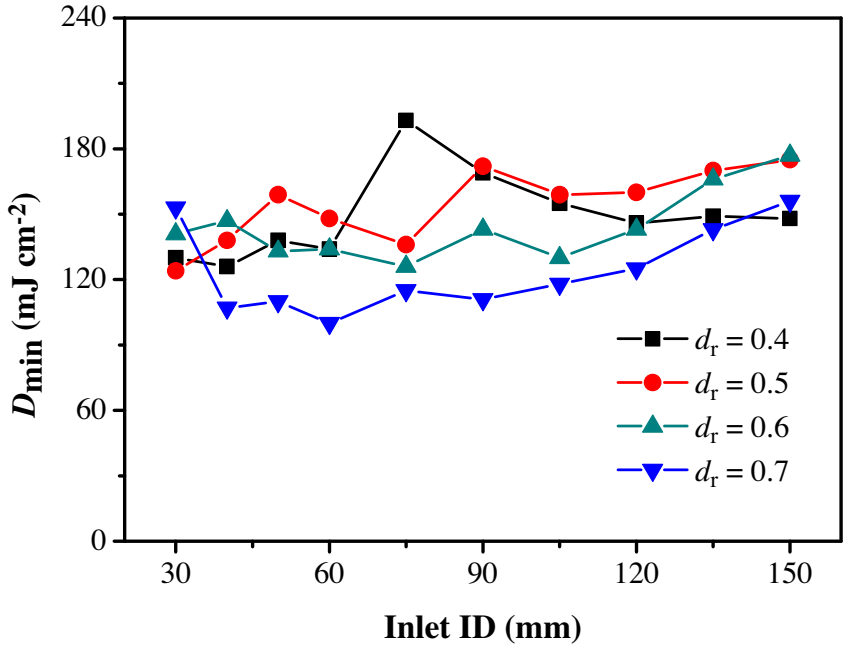

Fig. 4. Effects of the lamp relative distance $\left(d_{\mathrm{r}}\right)$ and inlet ID on the NOR UV reactor performance as indicated by $D_{\min }$.

Table 1

Characteristic parameters for FR distributions in the central cross sections of UV reactors with various lamp relative distances $\left(d_{\mathrm{r}}\right)$.

\begin{tabular}{lllll}
\hline$d_{\mathrm{r}}$ & 0.4 & 0.5 & 0.6 & 0.7 \\
\hline $\mathrm{FR}_{\mathrm{avg}}\left(\mathrm{mW} \mathrm{cm}^{-2}\right)$ & 32.9 & 32.3 & 30.9 & 28.8 \\
${ }^{*} \mathrm{SD}\left(\mathrm{mW} \mathrm{cm}^{-2}\right)$ & 26.6 & 22.5 & 20.6 & 21.8 \\
$\mathrm{RSD}$ & 0.781 & 0.672 & 0.641 & 0.795 \\
\hline
\end{tabular}

* Standard deviation.

ious $d_{\mathrm{r}}$ values. The area-average fluence rate $\left(\mathrm{FR}_{\mathrm{avg}}\right)$ decreased by $12 \%$ as the lamps moved closer to the reactor wall, that is, from $32.9 \mathrm{~mW} \mathrm{~cm}^{-2}$ for $d_{\mathrm{r}}=0.4-28.8 \mathrm{~mW} \mathrm{~cm}^{-2}$ for $d_{\mathrm{r}}=0.7$. This is reasonable because as the $d_{\mathrm{r}}$ increased, more UV radiation would be absorbed by the reactor wall. The lowest $\mathrm{FR}_{\mathrm{avg}}$ of reactors with $d_{\mathrm{r}}=0.7$ could partially explain their worst performances for most of the inlet IDs studied. Other two characteristic parameters for the cross-sectional radiation are the standard deviation of FR distribution and particularly the relative standard deviation (RSD). A smaller RSD means a more homogeneous FR distribution. For reactors with a large RSD, there usually exist some dark zones with a low FR, which tends to produce particles with a low UV dose. In fact, a dark zone close to the reactor wall could be observed in reactors with $d_{\mathrm{r}}=0.4(\mathrm{RSD}=0.781)$, and for reactors with $d_{\mathrm{r}}=0.7$ $(\mathrm{RSD}=0.795)$ the dark zones appeared in the near-wall region in between two lamps (Fig. S2). The relatively inhomogeneous FR distribution when the lamps were either close to each other or near the reactor wall was also observed by Li et al. [23], as supported by their in-situ measured FR data. The existence of dark zones was another reason for the poor performance of reactors with $d_{\mathrm{r}}=0.7$, and the relatively more homogeneous FR distribution in reactors with $d_{\mathrm{r}}=0.5$ generally led to a better performance.

As the inlet ID approached the reactor ID (i.e., $150 \mathrm{~mm}$ ), the flow field inside the reactor chamber became more uniform by forming a plug-like flow which induced the particles to travel in parallel (Fig. S3). In this situation, reactors with a more homogeneous FR distribution (i.e., with a lower RSD) should have a better performance. This explains the obviously better performances of reactors with $d_{\mathrm{r}}=0.5$ and 0.6 than those with $d_{\mathrm{r}}=0.4$ and 0.7 at an inlet ID of $150 \mathrm{~mm}$ (Fig. 4). As mentioned above, the largest $D_{\text {min }}$ was obtained for the NOR-0.4-75 reactor, which had an optimum combination of the lamp arrangement and inlet ID that well separated the influent flow with the lamp array and resulted in an 


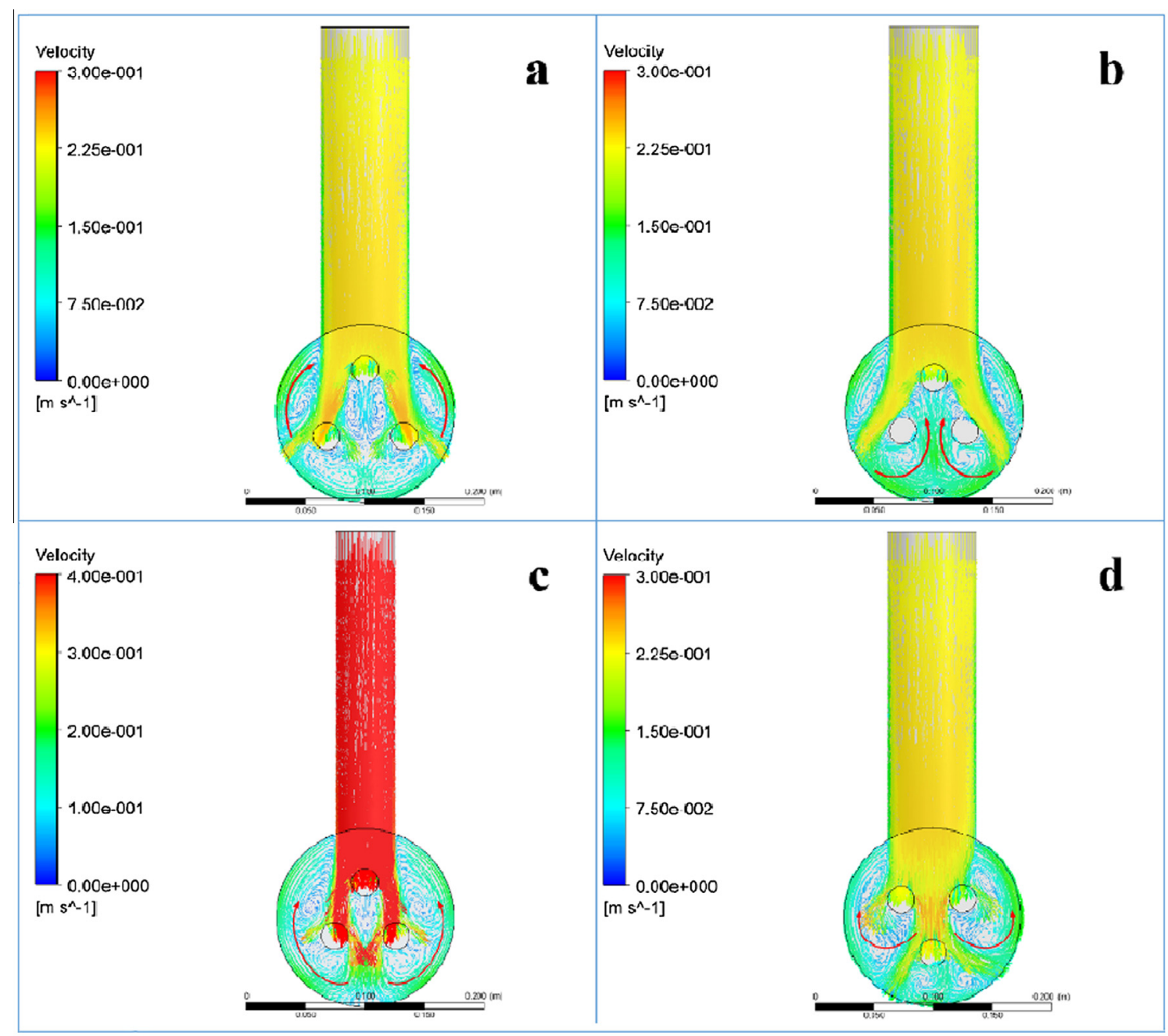

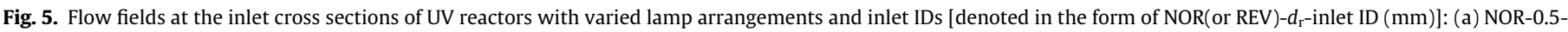
75, (b) NOR-0.4-75, (c) NOR-0.4-50, and (d) REV-0.4-75.

improved reactor performance. The effect of different combinations of the lamp arrangement and inlet ID on the reactor hydrodynamics was manifested by the flow fields at the inlet cross sections of UV reactors (Fig. 5). Compared to the flow whose main stream passed between the lamps in the reactor with the optimal configuration (NOR-0.4-75, Fig. 5b), the flows in reactors with a varied $d_{\mathrm{r}}$ (NOR-0.5-75, Fig. 5a) or inlet ID (NOR-0.4-50, Fig. 5c) swirled near the reactor wall. Considering the spatial FR distribution inside the reactor chamber, the first flow pattern was obviously favorable for a better reactor performance because it eliminated the probability of low UV dose particles.

\subsection{Effect of lamp position pattern on reactor performance}

The performances of UV reactors $(n=12)$ with different lamp position patterns (NOR or REV) and inlet IDs (50, 75 and $90 \mathrm{~mm}$ ) as indicated by $D_{\min }$ are shown in Fig. 6 . As the lamp position pattern was changed from NOR to REV, the corresponding $D_{\text {min }}$ decreased in most situations $(n=9)$ from the range of $110-193 \mathrm{~mJ} \mathrm{~cm}^{-2}$ to $98-160 \mathrm{~mJ} \mathrm{~cm}^{-2}$, with the greatest decline being more than $19 \%$. This is because when the lamps were positioned in the NOR pattern, the top lamp could act as a disperser for the influent flow to provide a better mixing (Fig. 5b), thus leading to a better reactor performance. The dispersion effect of the top lamp has also been reported by researchers working with 4-lamp

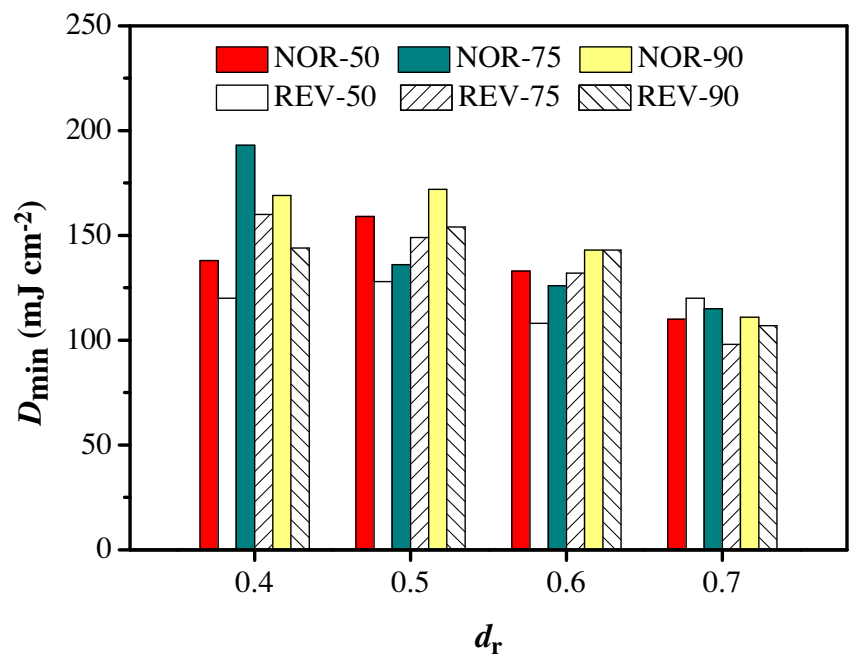

Fig. 6. Effect of lamp position pattern (NOR or REV) on UV reactor performance as indicated by $D_{\min }$ (inlet ID $=50,75$ and $90 \mathrm{~mm}$ ).

U-shape UV reactors [15]. Similar to the reactors with other nonoptimal configurations (i.e., NOR-0.5-75 and NOR-0.4-50), a swir- 
ling flow close to the reactor wall was observed in the REV reactor (REV-0.4-75, Fig. 5d), which was unfavorable for the reactor performance because of the dark zone in the near-wall region.

As can be seen from the above discussion, the FR distribution and hydrodynamics inside the reactor chamber had a complex effect on the reactor performance. This adds to the difficulty in UV reactor configuration optimization since the effects of reactor configuration on both the radiation and flow fields have to be considered. By using $D_{\min }$ as a performance indicator (that is, an increased $D_{\min }$ is beneficial for the reactor performance), the reactor configuration can be optimized more easily. The particle retention time with the minimum UV dose $\left(T_{\text {Dmin }}\right)$ in the configuration optimization simulations $(n=52)$ was recorded as well as the particle average retention time $\left(T_{\mathrm{avg}}\right)$ and the particle $10^{\text {th }}$ percentile retention time $\left(T_{10}\right)$ (Table $\left.S 1\right)$. It is quite surprising to find that a majority of the UV reactors $(n=35)$ had a $T_{\text {Dmin }}$ value greater than $T_{10}$, and the $T_{\text {Dmin }}$ of several UV reactors $(n=7)$ was even greater than $T_{\text {avg. }}$ This implies that in most cases the minimum UV dose was the result of a particle traveling for a long time in the dark zone rather than being flushed out of the reactor by a shortcircuiting flow. This finding is somewhat unexpected as shortcircuiting has always been considered as a primary cause for poor UV reactor performances [17,24]. From this respect, a well-mixed flow and a homogeneous FR distribution that prevent the longtime stay of a particle in the dark zone are the premises for a well-performing UV disinfection reactor. As to this study, the fact that the best reactor performance was found with the NOR-0.475 reactor demonstrates the great significance of a well-mixed flow, while the better performances of reactors with $d_{\mathrm{r}}=0.5$ reflect the importance of a homogeneous FR distribution.

\subsection{Determination of the maximum disinfection flow rate}

The objective of the configuration optimization of UV disinfection reactors is to elevate the $Q_{\max }$ so as to reduce the specific energy consumption. The $Q_{\max }$ can be determined by continuously increasing $Q$ until the corresponding UV dose decreases to a required value. For drinking water disinfection, the required minimum UV dose is $40 \mathrm{~mJ} \mathrm{~cm}^{-2}$ in China [16] and many other countries [5]; however, the designed RED of the UV reactor should be considerably higher considering the impacts of lamp aging and sleeve fouling. According to the national standard [16] and our previous study [4], the lamp aging, sleeve fouling and safety factors

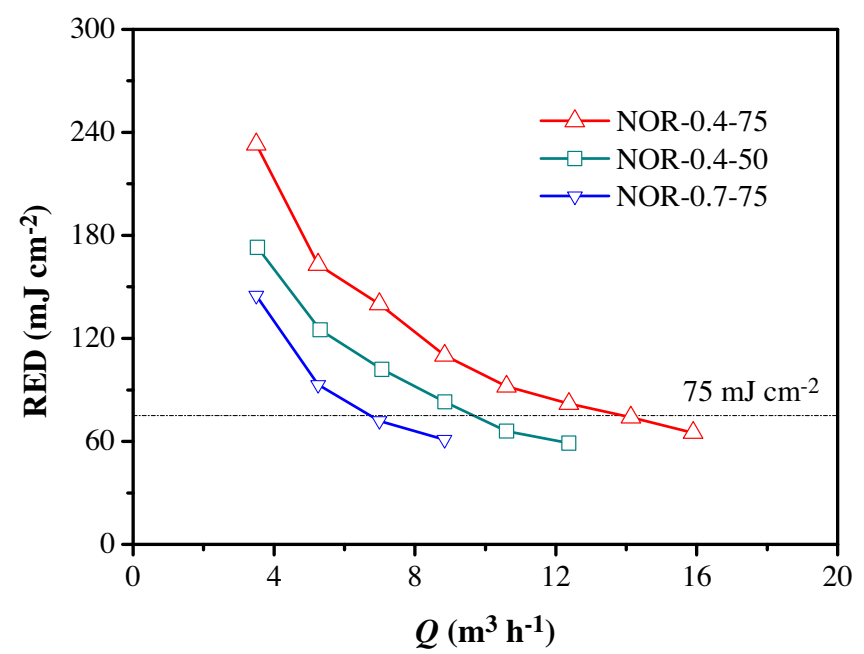

Fig. 7. RED-Q profiles of UV reactors with (NOR-0.4-75) or without (NOR-0.4-50 and NOR-0.7-75) the optimal configuration (for $Q_{\max }, R E D=75 \mathrm{~mJ} \mathrm{~cm}^{-2}$ ). were set at $0.8,0.8$ and 1.2 in this study, respectively. Hence, the $Q_{\max }$ of a UV disinfection reactor was determined when the corresponding RED reached $75 \mathrm{~mJ} \mathrm{~cm}^{-2}$. Following this criterion, the disinfection capabilities of three reactors with or without the optimal configuration were determined (Fig. 7). The NOR-0.4-75 reactor (i.e., the optimally designed reactor) could handle a $Q_{\max }$ of $14.0 \mathrm{~m}^{3} \mathrm{~h}^{-1}$, which was $47 \%$ higher than that of the NOR-0.4-50 reactor $\left(Q_{\max }=9.5 \mathrm{~m}^{3} \mathrm{~h}^{-1}\right)$ and $100 \%$ higher than that of the NOR-0.7-75 reactor $\left(Q_{\max }=7.0 \mathrm{~m}^{3} \mathrm{~h}^{-1}\right)$. It is clearly seen that the optimally designed UV reactor significantly reduced the specific energy consumption by elevating obviously the disinfection capability with the same lamp power input. To be specific, the NOR-0.475 reactor could save $32-50 \%$ of energy while still achieving the same disinfection efficiency.

Another important implication from Fig. 7 lies in that the UV reactor configuration optimization was valid over a wide $Q$ range as the RED- $Q$ profiles of the three reactors were approximately parallel. This finding is of great significance since it demonstrates the effectiveness of the reactor configuration optimization conducted with a certain $Q$ and avoids a lot of repetitive work in verifying the optimization results for many other different conditions.

\section{Conclusions}

This study proposed a new and simple performance indicator, $D_{\text {min }}$, for UV reactors for water disinfection and examined its feasibility in characterizing the reactor performance through optimizing the configuration of 3-lamp UV disinfection reactors. The effects of the lamp arrangement and inlet size on the reactor performance were also investigated through analysis of the FR distribution and hydrodynamics inside the reactor chamber. In addition, the $Q_{\max }$ values of reactors with or without the optimal configuration were comparatively determined to assess the energy saving efficiency. Based on the experimental results, the main conclusions can be drawn as follows:

- $D_{\text {min }}$ can serve as a qualified performance indicator in place of RED for UV disinfection reactors, as demonstrated by both theoretical deductions and CFD simulations.

- In general, the NOR reactors had larger $D_{\text {min }}$ values $\left(110-193 \mathrm{~mW} \mathrm{~cm}^{-2}\right)$ than the $\mathrm{REV}$ reactors (98-160 $\left.\mathrm{mW} \mathrm{cm}^{-2}\right)$. The largest $D_{\min }\left(193 \mathrm{~mW} \mathrm{~cm}^{-2}\right)$ was obtained in the NOR-0.4-75 reactor because of a well-mixed flow resulting from an optimal dispersion of the influent flow by the lamp array.

- The $Q_{\max }$ of the optimally designed UV reactor (i.e., NOR-0.4-75) was elevated by $47-100 \%$ compared to those of the nonoptimally designed reactors, which could save $32-50 \%$ of energy.

- The proposed new performance indicator for UV disinfection reactors, $D_{\min }$, has distinct advantages over the commonlyused RED in being independent of the microbial doseresponse curve as well as free of switching back and forth between fluence and survival microbial concentration. Hence, replacing RED with $D_{\min }$ as a performance indicator can not only optimize the UV reactor configuration more efficiently, but also reduce the design error considerably.

\section{Acknowledgments}

The authors gratefully acknowledge the financial support from the National Natural Science Foundation (51290281, 51525806, 21590814) and the Ministry of Science and Technology (2012ZX07404-004) of China. 


\section{Appendix A. Supplementary data}

Supplementary data associated with this article can be found, in the online version, at http://dx.doi.org/10.1016/j.cej.2016.07.042.

\section{References}

[1] W.A.M. Hijnen, E.F. Beerendonk, G.J. Medema, Inactivation credit of UV radiation for viruses, bacteria and protozoan (oo) cysts in water: a review, Water Res. 40 (2006) 3-22.

[2] F.A. Momani, C. Sans, S. Esplugas, A comparative study of the advanced oxidation of 2,4-dichlorophenol, J. Hazard. Mater. B107 (2004) 123-129.

[3] L. Oin, Y.L. Lin, B. Xu, C.Y. Hu, F.X. Tian, T.Y. Zhang, W.O. Zhu, H. Huang, N.Y. Gao, Kinetic models and pathways of ronidazole degradation by chlorination, UV irradiation and UV/chlorine processes, Water Res. 65 (2014) 271-281.

[4] M.K. Li, Z.M. Qiang, J.R. Bolton, W.T. Li, P. Chen, UV disinfection of secondary water supply: Online monitoring with micro-fluorescent silica detectors, Chem. Eng. J. 255 (2014) 165-170.

[5] USEPA, Ultraviolet Disinfection Guidance Manual for the Final Long Term 2 Enhanced Surface Water Treatment Rule (EPA 815-R-06-007), Office of Water, U.S. Environmental Protection Agency, Washington DC, 2006.

[6] E.R. Blatchley III, C.Y. Shen, O.K. Scheible, J.P. Robinson, K. Ragheb, D.E. Bergstrom, D. Rokjer, Validation of large-scale, monochromatic UV disinfection systems for drinking water using dyed microspheres, Water Res. 42 (2008) 677-688.

[7] F. Solari, G. Girolimetti, R. Montanari, G. Vignali, A new method for the validation of ultraviolet reactors by means of photochromic materials, Food Bioprocess. Technol. 8 (2015) 2192-2211.

[8] T. Sultan, Numerical study of the effects of lamp configuration and reactor wall roughness in an open channel water disinfection UV reactor, Chemosphere 155 (2016) 170-179.

[9] D.A. Sozzi, F. Taghipour, UV reactor performance modeling by Eulerian and Lagrangian methods, Environ. Sci. Technol. 40 (2006) 1609-1615.

[10] C.K. Ho, S.S. Khalsa, H.B. Wright, E. Wicklein, Modeling UV disinfection using integrated computational fluid dynamics and discrete ordinates radiation models, Proceedings of Disinfection 2009, Water Environment Federation, Alexandria, VA, 2009, pp. 257-273.

[11] J.J. Ducoste, D. Liu, K. Linden, Alternative approaches to modeling fluence distribution and microbial inactivation in ultraviolet reactors: Lagrangian versus Eulerian, J. Environ. Eng. 131 (2005) 1393-1403.
[12] B.A. Wols, C.H.M. Hofman-Caris, D.J.H. Harmsen, E.F. Beerendonk, J.C. van Dijk, P. Chan, E.R. Blatchley III, Comparison of CFD, biodosimetry and Lagrangian actinometry to assess UV reactor performance, Ozone Sci. Eng. 34 (2012) 8191.

[13] A. Cabaj, R. Sommer, D. Schoenen, Biodosimetry: model calculations for u.v. water disinfection devices with regard to dose distributions, Water Res. 30 (1996) 1003-1009.

[14] B.A. Wols, J.A.M.H. Hofman, E.F. Beerendonk, W.S.J. Uijttewaal, J.C. van Dijk, A systematic approach for the design of UV reactors using computational fluid dynamics, AIChE J. 57 (2011) 193-207.

[15] C. Xu, X.S. Zhao, G.P. Rangaiah, Performance analysis of ultraviolet water disinfection reactors using computational fluid dynamics simulation, Chem. Eng. J. 221 (2013) 398-406.

[16] Chinese National Standardization Management Committee, Ultraviolet (UV) Disinfection Equipment for Municipal Water and Wastewater Treatment, GB/T 19837-2005, 2005. (In Chinese).

[17] J. Zhang, A.E. Tejada-Martnez, Q. Zhang, Developments in computational fluid dynamics-based modeling for disinfection technologies over the last two decades: a review, Environ. Model. Softw. 58 (2014) 71-85.

[18] D.A. Sozzi, F. Taghipour, Computational and experimental study of annular photo-reactor hydrodynamics, Int. J. Heat Fluid Flow 27 (2006) 1043-1053.

[19] C.K. Ho, Evaluation of reflection and refraction in simulations of ultraviolet disinfection using the discrete ordinates radiation model, Water Sci. Technol. 59 (2009) 2421-2428.

[20] J.E. Duran, F. Taghipour, M. Mohseni, Irradiance modeling in annular photoreactors using the finite-volume method, J. Photochem. Photobio. A: Chem. 215 (2010) 81-89.

[21] J.J. Ducoste, D. Liu, K.G. Linden, H. Mamane-Gravetz, Z. Bohrerova, Impact of influent pipe configuration on UV reactor performance. Is the elbow truly the worst case hydraulic condition?, Proceedings of Disinfection 2005, Water Environment Federation, Alexandria, VA, 2005, pp 257-270.

[22] A. Munoz, S. Craik, S. Kresta, Computational fluid dynamics for predicting performance of ultraviolet disinfection-sensitivity to particle tracking inputs, J. Environ. Eng. Sci. 6 (2007) 285-301.

[23] M.K. Li, Z.M. Qiang, J.R. Bolton, In situ detailed fluence rate distributions in a UV reactor with multiple low-pressure lamps: comparison of experimental and model results, Chem. Eng. J. 214 (2013) 55-62.

[24] F. Taghipour, A. Sozzi, Modeling and design of ultraviolet reactors for disinfection by-product precursor removal, Desalination 176 (2005) 71-80. 